\title{
Topological dilaton black holes
}

\author{
Rong-Gen Cai \\ Center for Theoretical Physics, Seoul National University, Seoul 151-742, Korea \\ Jeong-Young Ji and Kwang-Sup Soh \\ Department of Physics Education, Seoul National University, Seoul 151-742, Korea
}

\begin{abstract}
In four-dimensional spacetime, when the two-sphere of black hole event horizons is replaced by a two-dimensional hypersurface with zero or negative constant curvature, the black hole is referred to as a topological black hole. In this paper we present some exact topological black hole solutions in the EinsteinMaxwell-dilaton theory with a Liouville-type dilaton potential.
\end{abstract}

PACS numbers: 04.20.Jb, 04.20.Gz, 04.70.Dy 
The topological structure of the event horizon of a black hole is an intriguing subject in black hole physics. It is generally believed that a black hole in the four dimensional spacetime is always with a spherical topology. That is, the event horizon of a black hole has the topology $S^{2}$. This was proven by Friedman, Schleich and Witt [1]. They suggested the "topological censorship theorem", which states that in a globally hyperbolic, asymptotically flat spacetime satisfying the null energy condition, every causal curve (nonspacelike curve) from $\mathcal{J}^{-}$to $\mathcal{J}^{+}$is homotopic to a topologically trivial curve from $\mathcal{J}^{-}$to $\mathcal{J}^{+}$. That is, general relativity does not allow an observer to probe the topology of spacetime: Any topological structure collapses too quickly to allow light to traverse it. Later on, however, they found that nontrivial topologies can be observed passively [2]. The black holes with toroidal topology have indeed been found numerically in the gravitational collapse [3], although such a topological structure is temporal.

When the asymptotic flatness and the energy condition are given up, there are no fundamental reasons to forbid the existence of static or stationary black holes with nontrivial topologies. In particular, when the spacetime is asymptotically anti-de Sitter one, the matter field can be in stable equilibrium even if the potential energy is unbounded from below. In recent years, there has been a growing interest in these black holes with nontrivial topological structures (topological black holes) in the asymptotically anti-de Sitter space [1 [13]. These investigations are mainly based on the Einstein (-Maxwell) theory with a negative cosmological constant. In general, one has the static solutions of Einstein-Maxwell equations with a cosmological constant

$$
d s^{2}=-\left(k-\frac{2 M}{r}+\frac{Q^{2}}{r^{2}}-\frac{1}{3} \Lambda r^{2}\right) d t^{2}+\left(k-\frac{2 M}{r}+\frac{Q^{2}}{r^{2}}-\frac{1}{3} \Lambda r^{2}\right)^{-1} d r^{2}+r^{2} d \Omega_{k}^{2},
$$

where $d \Omega_{k}^{2}$ is the line element of a two-dimensional hypersurface $\Sigma$ with constant curvature,

$$
d \Omega_{k}^{2}=\left\{\begin{array}{lll}
d \theta^{2}+\sin ^{2} \theta d \phi^{2}, & \text { for } k=1 \\
d \theta^{2}+\theta^{2} d \phi^{2}, & \text { for } k=0 \\
d \theta^{2}+\sinh ^{2} \theta d \phi^{2}, & \text { for } k=-1 .
\end{array}\right.
$$

Here $M$ and $Q$ are the mass and charge of the solution, respectively, and the cosmological constant $\Lambda$ is negative. For $k=1$, the metric (1) describes the spacetime of the ReissnerNordström-anti-de Sitter black holes. Here the event horizon of the black hole has the 2 -sphere topology $S^{2}$, and the topology of spacetime is $R^{2} \times S^{2}$. For $k=0$, if we identify the coordinates $\theta$ and $\phi$ with certain periods, the topology of event horizon is that of a torus and the spacetime has the topology $R^{2} \times T^{2}$. For $k=-1$, the surface $\Sigma$ is a 2-dimensional hypersurface with constant negative curvature. The topology of spacetime is $R^{2} \times H_{g}^{2}$ [9], where $H_{g}^{2}$ is the topology of the surface $\Sigma$. Brill et al. [12] have discussed in detail the topological structure of solution (四). In the case of $k=-1$, the solution (11) is of some strange properties. Usually the occurrence of black hole horizon is always related to the positive definiteness of energy of spacetime. In Eq. (1), however, even if $M=Q=0$, one has the black hole structure with black hole horizon $r_{h}=\sqrt{3 /|\Lambda|}$. In particular, when the mass is negative, one still has the black hole solution. Surprisingly, this negative mass black hole can also be formed by gravitational collapse [11]. Obviously, the asymptotically anti-de Sitter behavior plays a crucial role in the existence of these nontrivial topological black holes. 
Note that only in Ref. [6] one of the present authors and Zhang have considered briefly the dilaton black plane solutions $(k=0)$. In the present paper we would like to investigate the deformation of these topological black holes (11) by a dilaton field and a Liouville-type dilaton potential (an effective cosmological constant). Due to the dilaton field, the topological dilaton black holes found here will become neither asymptotically anti-de Sitter nor asymptotically flat.

Consider the following action

$$
S=\frac{1}{16 \pi} \int d^{4} x \sqrt{-g}\left[R-2(\nabla \phi)^{2}-2 \Lambda e^{2 b \phi}-e^{-2 a \phi} F_{\mu \nu} F^{\mu \nu}\right],
$$

where $a$ and $b$ are two constants and $F_{\mu \nu}$ is the Maxwell field. We still refer to $\Lambda$ as the "cosmological constant". This action (3) has been investigated in some detail by Chan et al. [14] in finding the spherically symmetric black holes. So in this paper we will consider the cases of $k=0$ and $k=-1$ only. Varying the action (3) yields the equations of motion

$$
\begin{aligned}
& R_{\mu \nu}=2 \partial_{\mu} \phi \partial_{\nu} \phi+g_{\mu \nu} \Lambda e^{2 b \phi}+2 e^{-2 a \phi}\left(F_{\mu \lambda} F_{\nu}{ }^{\lambda}-\frac{1}{4} g_{\mu \nu} F^{2}\right), \\
& 0=\partial_{\mu}\left(\sqrt{-g} e^{-2 a \phi} F^{\mu \nu}\right), \\
& \nabla^{2} \phi=b \Lambda e^{2 b \phi}-\frac{a}{2} e^{-2 a \phi} F^{2} .
\end{aligned}
$$

We assume the metric to be solved being of the form

$$
d s^{2}=-U(r) d t^{2}+U^{-1}(r) d r^{2}+R^{2}(r) d \Omega_{k}^{2} .
$$

Thus Eq. (5) can be easily integrated to obtain

$$
F_{t r}=\frac{4 \pi Q}{V R^{2}} e^{2 a \phi} .
$$

Here $V$ is the area of the hypersurface $\Sigma$ when it is closed, and $Q$ is the electric charge defined as $Q=-\frac{1}{8 \pi} \int e^{-2 a \phi} \varepsilon_{\mu \nu \alpha \beta} F^{\alpha \beta}$. The equations (四) and (6) then can be simplified to

$$
\begin{aligned}
& -\frac{U^{\prime \prime}}{2}-\frac{R^{\prime} U^{\prime}}{R}=\Lambda e^{2 b \phi}-\frac{16 \pi^{2} Q^{2}}{V^{2} R^{4}} e^{2 a \phi}, \\
& -\frac{U^{\prime \prime}}{2}-\frac{R^{\prime} U^{\prime}}{R}-\frac{2 R^{\prime \prime} U}{R}=2 U \phi^{\prime 2}+\Lambda e^{2 b \phi}-\frac{16 \pi^{2} Q^{2}}{V^{2} R^{4}} e^{2 a \phi}, \\
& -\frac{1}{2 R^{2}}\left[U\left(R^{2}\right)^{\prime}\right]^{\prime}+\frac{k}{R^{2}}=\Lambda e^{2 b \phi}+\frac{16 \pi^{2} Q^{2}}{V^{2} R^{4}} e^{2 a \phi}, \\
& \frac{1}{R^{2}}\left[R^{2} U \phi^{\prime}\right]^{\prime}=\Lambda b e^{2 b \phi}+\frac{16 \pi^{2} a Q^{2}}{V^{2} R^{4}} e^{2 a \phi},
\end{aligned}
$$

where a prime represents the derivative with respect to $r$. From Eqs. (9) and (10) we have

$$
R^{\prime \prime} / R=-\phi^{\prime 2}
$$

We further assume

$$
R(r)=\gamma r^{N}
$$


where $\gamma$ and $N$ are two constants. Such an assumption (14) has been extensively used to look for the dilaton black hole solutions [14,15]. Thus from (13) one has

$$
\phi(r)=\phi_{0} \pm \sqrt{N(1-N)} \ln r
$$

where $\phi_{0}$ is an integration constant. We now discuss the cases of $k=0$ and $k=-1$, respectively.

(I) $k=0$ : In this case we find two sets of solutions of physical interest. The first set is

$$
\begin{aligned}
& U(r)=-\frac{8 \pi M}{V N \gamma^{2}} r^{1-2 N}-\frac{\Lambda e^{2 b \phi_{0}}}{N(4 N-1)} r^{2 N}+\frac{16 \pi^{2} Q^{2} e^{2 a \phi_{0}}}{N V^{2} \gamma^{4}} r^{-2 N}, \\
& \phi(r)=\phi_{0}+\sqrt{N(1-N)} \ln r \\
& a=b=\sqrt{N(1-N)} / N
\end{aligned}
$$

where $M$ is the quasilocal mass defined as in Refs. 16].15]. If $a=b=-\sqrt{N(1-N)} / N$, the solution (16) remains unchanged, but $\phi(r)$ becomes $\phi(r)=\phi_{0}-\sqrt{N(1-N)} \ln r$. From (18) we must have $0<N<1$ (as $N=1$ one has only the trivial constant dilaton solution), and $N \neq 1 / 4$ [see Eq. (16)] .

(i) $0<N<1 / 4$ : The first term $\left(r^{1-2 N}\right)$ in (16) is dominant as $r$ is very large. That is, $U(r)$ is negative as $r$ is enough large. In this case, the solution (16) will have a cosmological horizon, despite the sign of the cosmological constant $\Lambda$. The horizons are determined by

$$
-\frac{8 \pi M}{V \gamma^{2}} r-\frac{\Lambda e^{2 b \phi_{0}}}{(4 N-1)} r^{4 N}+\frac{16 \pi^{2} Q^{2} e^{2 a \phi_{0}}}{V^{2} \gamma^{4}}=0,
$$

and due to $4 N<1$, this equation has only a positive real root, which corresponds to the cosmological horizon. The Hawking temperature of the horizon is

$$
T_{h}=\left|-\frac{2 M}{V N \gamma^{2}} r_{c}^{-2 N}-\frac{\Lambda e^{2 b \phi_{0}}}{(4 N-1) \pi} r_{c}^{2 N-1}\right|,
$$

where $r_{\mathrm{c}}$ is the cosmological horizon.

(ii) $1 / 4<N<1$ : The second term $\left(r^{2 N}\right)$ in the solution (16) will be dominant as $r \rightarrow \infty$. Due to $4 N>1$, equation (19) has at most two positive roots. For $\Lambda>0$, equation (19) clearly has only one solution, which corresponds to the cosmological horizon. For $\Lambda<0$, the Eq. (19) has no cosmological horizon. But it may have the black hole horizons which are still determined by the equation (19). For example, for $N=1 / 2$, we have black hole horizons

$$
r_{ \pm}=\frac{4 \pi M}{V \gamma^{2}|\Lambda| e^{2 \phi_{0}}}\left[1 \pm \sqrt{1-\frac{Q^{2}|\Lambda| e^{4 \phi_{0}}}{M^{2}}}\right] .
$$

For $M^{2}>|\Lambda| e^{4 \phi_{0}} Q^{2}$, the solution (16) has two black hole horizons, outer horizon $r_{+}$and inner horizon $r_{-}$. For $M^{2}=|\Lambda| Q^{2} e^{4 \phi_{0}}$, the two horizons coincide. This corresponds to the extremal topological dilaton black hole. For $M^{2}<|\Lambda| Q^{2} e^{4 \phi_{0}}$, the singularity at $r=0$ will be naked. Analytically continuing the black hole solution to its Euclidean section, it is easy 
to find the Hawking temperature of the hole by requiring the absence of conical singularity at the black hole horizon. The Hawking temperature of the black hole is found to be

$$
T_{h}=\frac{|\Lambda| e^{2 \phi_{0}}}{2 \pi}-\frac{8 \pi Q^{2} e^{2 \phi_{0}}}{V^{2} \gamma^{4} r_{+}^{2}} .
$$

It is worth noting that when $Q=0$, the Hawking temperature will become a constant independent of the mass. This property is very similar to that of the 2-dimensional charged dilaton black holes [17]. For $N=3 / 4$, the solution (16) may also have two black hole horizons. We can obtain similar expressions of black hole horizons and the Hawking temperature as well.

The second set of solutions is

$$
\begin{aligned}
& U(r)=-\frac{8 \pi M}{V N \gamma^{2}} r^{1-2 N}+\frac{32 \pi^{2} N Q^{2} e^{2 a \phi_{0}}}{V^{2} \gamma^{4} x(x+b N)(2 b x+2 N+1)} r^{2+2 b x}, \\
& \phi(r)=\phi_{0}+x \ln r \\
& (a-b) x=2 N \\
& \Lambda=-\frac{x+a N}{x+b N} \frac{16 \pi^{2} Q^{2} e^{2(a-b) \phi_{0}}}{V^{2} \gamma^{4}},
\end{aligned}
$$

where $x=\sqrt{N(1-N)}$. The solution (23) has three possibilities: naked singularity, a cosmological horizon or a black hole horizon, depending on the parameters $a$ and $b$. When the horizon is cosmological one, the singularity at $r=0$ is a cosmological one. The black hole horizon is

$$
r_{+}=\left[\frac{4 \pi N^{2} Q^{2} e^{2 a \phi_{0}}}{V \gamma^{2} x(x+b N)(2 b x+2 N+1) M}\right]^{-1 /(1+2 N+2 b x)},
$$

and the associated Hawking temperature is

$$
T_{h}=\frac{2(2 N-1) M}{V N \gamma^{2} r_{+}^{2 N}}+\frac{16 \pi N(1+b x) N Q^{2} e^{2 a \phi_{0}} r_{+}^{1+2 b x}}{V^{2} \gamma^{4} x(x+b N)(2 b x+2 N+1)} .
$$

(II) $k=-1:$ In this case, we also find two sets of solutions of physical interest. The first is

$$
\begin{aligned}
& U(r)=-\frac{8 \pi M}{V N \gamma^{2}} r^{1-2 N}-\frac{\Lambda e^{2 b \phi_{0}}}{(1-N)} r^{2-2 N}+\frac{16 \pi^{2} Q^{2} e^{2 a \phi_{0}}}{N V^{2} \gamma^{4}} r^{-2 N} \\
& \phi(r)=\phi_{0}-\sqrt{N(1-N)} \ln r \\
& b=a^{-1}=N / \sqrt{N(1-N)}, \\
& \Lambda=-\frac{1-N}{1-2 N} \frac{e^{-2 b \phi_{0}}}{\gamma^{2}} .
\end{aligned}
$$

If $b=a^{-1}=-N / \sqrt{N(1-N)}$, the solution (29) remains unchanged, but $\phi(r)=\phi_{0}+$ $\sqrt{N(1-N)} \ln r$. When $\Lambda>0$ and $1 / 2<N<1$ [see Eq. (32)], the solution (29) has only one cosmological horizon 


$$
r_{\mathrm{c}}=\frac{4 \pi(1-N) M}{V N \Lambda e^{2 b \phi_{0}}}\left[-1+\sqrt{1+\frac{N \Lambda Q^{2} e^{2(a+b) \phi_{0}}}{(1-N) M^{2}}}\right] .
$$

When $\Lambda<0$ and $0<N<1 / 2$, we have two black hole horizons

$$
r_{ \pm}=\frac{4 \pi(1-N) M}{V N|\Lambda| \gamma^{2} e^{2 b \phi_{0}}}\left[1 \pm \sqrt{1-\frac{N|\Lambda| Q^{2} e^{2(a+b) \phi_{0}}}{(1-N) M^{2}}}\right]
$$

and the Hawking temperature is

$$
T_{h}=\frac{2(2 N-1) M}{V N \gamma^{2} r_{+}^{2 N}}+\frac{|\Lambda| e^{2 b \phi_{0}} r_{+}^{1-2 N}}{2 \pi}-\frac{8 \pi Q^{2} e^{2 a \phi_{0}}}{V^{2} \gamma^{4} r_{+}^{2 N+1}}
$$

The second set of solutions is

$$
\begin{aligned}
& U(r)=-\frac{8 \pi M r^{1-2 N}}{V N \gamma^{2}}-\frac{\Lambda e^{2 b \phi_{0}} r^{2-2 N}}{(1-N)}+\frac{16 \pi^{2} Q^{2} e^{2 a \phi_{0}} r^{2-2 N}}{V^{2} \gamma^{4}(1-N)}, \\
& \phi(r)=\phi_{0}+\sqrt{N(1-N)}, \\
& a=-b=N / \sqrt{N(1-N)}, \\
& \Lambda=-\frac{(1-N) e^{-2 b \phi_{0}}}{\gamma^{2}(1-2 N)}\left[\frac{16 \pi^{2} Q^{2} e^{2 a \phi_{0}}}{V^{2} \gamma^{2}(1-N)}+1\right],
\end{aligned}
$$

When $a=-b=-N / \sqrt{N(1-N)}$, the solution $U(r)$ keeps unchanged, but $\phi(r)=\phi_{0}-$ $\sqrt{N(1-N)} \ln r$. When $\Lambda<0$, namely, $0<N<1 / 2$ [see Eq. (39)], obviously, the solution (36) has a black hole horizon

$$
r_{+}=\frac{8 \pi M}{V N \gamma^{2}}\left[-\frac{\Lambda e^{2 b \phi_{0}}}{(1-N)}+\frac{16 \pi^{2} Q^{2} e^{2 a \phi_{0}}}{V^{2} \gamma^{4}(1-N)}\right]^{-1} .
$$

The Hawking temperature is

$$
T_{h}=\frac{2(2 N-1) M}{V N \gamma^{2} r_{+}^{2 N}}-\frac{\Lambda e^{2 b \phi_{0}} r_{+}^{1-2 N}}{2 \pi}+\frac{8 \pi Q^{2} e^{2 a \phi_{0}} r_{+}^{1-2 N}}{V^{2} \gamma^{4}} .
$$

These topological dilaton black hole solutions are counterparts of the spherically symmetric black holes $(k=1)$ [14]. They are neither asymptotically flat nor (anti-)de Sitter, but they have finite quasilocal masses and finite conserved charge for compact event horizons. These solutions have only a singularity at $r=0$, and is enclosed by black hole horizons or cosmological horizon. In addition, although these topological dilaton black holes exhibit unusual asymptotic behavior, it is easy to show that the entropy of black holes still obeys the area formula:

$$
S=\frac{1}{4} V R^{2}\left(r_{+}\right)=\frac{1}{4} A\left(r_{+}\right),
$$

where $A\left(r_{+}\right)=V R^{2}\left(r_{+}\right)$is the horizon area of topological black holes. This is because the black hole entropy comes from the surface term of the Euclidean counterpart of action (3) 
and the entropy is just the value of the surface term at the black hole horizon 18. In the above we only analyze the causal structure of solutions for the positive quasilocal mass. If a negative quasilocal mass is allowed, there are richer black hole structures for both cases of $k=0$ and $k=1$. Similar to that in Ref. [14], the generalization of the above topological dilaton black holes to two Liouville-type potential $V=2 \Lambda_{1} e^{2 b_{1} \phi}+2 \Lambda_{2} e^{2 b_{2} \phi}\left(b_{1} \neq b_{2}\right)$ is a straightforward work, but the causal structure of solutions will become complicated. Due to the dilaton black hole solutions obtained by Chan et al. [14], our black hole solutions presented here can be regarded as complements of those spherically symmetric dilaton black holes. Finally, it is worth noting that when $\Lambda=0$, the action (3) allows the existence of the spherically symmetric black hole solutions, but does not for the topological dilaton black holes.

In summary, we have obtained some topological black hole solutions in the dilaton gravity with a Liouville-type dilaton potential. Their event horizons are the hypersurfaces with zero curvature or negative constant curvature. From these solutions we have found that the effective cosmological constant in the action (3) must still be negative in order to have these topological black hole solutions. This situation is quite different from that of the spherically symmetric dilaton black holes [14].

This work was supported by the Center for Theoretical Physics of Seoul National University. 


\section{REFERENCES}

[1] J. L. Friedman, K. Schleich, and D. M. Witt, Phys. Rev. Lett. 71, 1486 (1993).

[2] J. L. Friedman, K. Schleich, and D. M. Witt, Phys. Rev. Lett. 75, 1872 (1995).

[3] S. L. Shapiro, S. A. Teukolsky, and J. Winicour, Phys. Rev. D 52, 6982 (1995).

[4] J. P. S. Lemos, Class. Quantum Grav. 12, 1081 (1995); Phys. Lett B 353, 46 (1996); J. P. S. Lemos and V. T. Zanchin, Phys. Rev. D 54, 3840 (1996).

[5] C. G. Huang and C. B. Liang, Phys. Lett. A 201, 27 (1995); C. G. Huang, Acta Phys. Sin. 4, 617 (1996).

[6] R. G. Cai and Y. Z. Zhang, Phys. Rev. D 54, 4891 (1996).

[7] S. Aminneborg, I. Bengtsson, S. Holst, and P. Peldan, Class. Quantum Grav. 13, 2707 (1996).

[8] R. B. Mann, Class. Quantum Grav.14, L109 (1997); "Charged topological black hole pair creation", Report hep-th/9705223.

[9] W. L. Smith and R. B. Mann, Phys. Rev. D 56, 4942 (1997).

[10] M. Bañados, "Constant Curvature Black Holes", Report gr-qc/9703040.

[11] R. B. Mann, Class. Quantum Grav. 14, 2927 (1997).

[12] D. R. Brill, J. Louko, and P. Peldan, Phys. Rev. D 56, 3600 (1997).

[13] L. Vanzo, Phys. Rev. D 56, 6475 (1997).

[14] K. C. K. Chan, J. H. Horne, and R. B. Mann, Nucl. Phys. B 447, 441 (1995).

[15] K. C. K. Chan and R. B. Mann, Phys. Rev. D 50, 6385 (1994) ; Phys. Lett. B 371, 199 (1996); K. C. K. Chan, Phys. Rev. D 55, 3564 (1997).

[16] J. D. Brown and J. W. York, Phys. Rev. D 47, 1407 (1993); J. D. Brown, J. Creighton, and R. B. Mann, Phys. Rev. D 50, 6394 (1994).

[17] V. P. Frolov, Phys. Rev. D 46, 5383 (1992).

[18] R. G. Cai and Y. S. Myung, Phys. Rev. D 56, 3466 (1997). 